by the sulphuric acid method and the direct ignition process, the figures for the same sample not being at all concordant.

A constant weight was not obtainable by this process and the composition of the ash varied considerably with the length of time of the incineration and the temperature employed.

At somewhat elevated temperatures it was found that chlorides were more or less attacked and, possibly, partly volatilized.

ALBERTI AND HEMPEL'S METHOD FOR ASH

DETERMINATIONS.

Stift (Oest. Ztschr. Zucker, 22, 22; Chem. Ztg. Rep., 17, 102), as the result of a large number of experiments with different sugar products, concludes that this method, by proper and careful execution, and with previous thorough testing of the quartz sand employed, is to be most highly commended, yielding, as it does, concordant results and, in contrast to other methods, furnishing more correct and uniform figures for the content of inorganic substances.

A point of great importance in the performance of the incineration is the necessity of the employment of quartz sand of absolute and known purity.

With sugars containing considerable proportions of nitrates (which are decomposed in this process), it is necessary to make a separate estimation of nitric acid.

\title{
THE DETERMINATION OF VOLATILE AND INSOLUBLE FATTY ACIDS IN BUTTER FAT.
}

\author{
BY W. H. BEAL.
}

Received July 27,2894

\begin{abstract}
$A$ FEW years ago when called upon to make examinations of $A$ the fat in a number of samples of cream the author attempted to use for that purpose the then recently proposed method of Waller as modified by Moore' which is briefly as follows: Two and five-tenths grams of fat are placed in a weighed Erlenmeyer flask and saponified with one gram of potassium hydroxide dissolved in fifty cc. of seventy per cent. alcohol. After heating until saponification is complete the alcohol is driven off in a boiling water-bath. The resulting soap is dissolved in fifty cc.

1 See $J$. Am. Chem. Soc., I1, I44, 1889.
\end{abstract}


of water and decomposed by means of twenty $c c$. of dilute sulphuric acid (one part of strong acid to ten parts of water). The flask is connected with a condenser and fifty cc. are distilled off, the distillate passing through a small filter in the mouth of the receiving flask. The distillate is titrated with one-tenth normal sodium hydroxide solution, using phenolphthalein as an indicator. Fifty cc. of water are added to the contents of the flask and an equal quantity distilled off and titrated as before. This treatment is repeated until only one-tenth cc. or less of soda solution is required to neutralize the distillate. After cooling, the liquid remaining in the flask is poured off from the solidified insoluble acids through the same filter used for the distillates. The insoluble acids are repeatedly washed with hot water until all traces of sulphuric acids are removed. The condenser is then washed out with hot alcohol and the filter exhausted with the same solvent, the washings being collected in the flask containing the insoluble acids. The alcohol is driven off and the flask and contents dried at $100^{\circ} \mathrm{C}$. and weighed.

With proper precautions satisfactory results were obtained with this method, but the work had not proceeded far before grounds for the following objections became evident: (I) Saponification is slow and removal of the last traces of alcohol in the dilute solution recommended is difficult; (2) there is danger from violent bumping, which it was found impossible to entirely prevent; (3) there is danger of charring the fat adhering to the sides of the flask when the contents of the latter are reduced to a small bulk, causing the final insoluble acid to have a brownish color instead of a pale yellow color which is normal; and (4) inconvenience and loss of time is occasioned by the necessity of frequently replenishing the contents of the flask.

An attempt to devise a process which would in a measure, at least, overcome these difficulties, which it may be observed are in large part shared by all the methods now in vogue, resulted finally in the adoption of the following method of procedure:

Volatile Acids. - Two and eight-tenths to two and nine-tenths cc. (approximately two and five-tenths grams) of the pure fat were measured by means of a narrow pipette into a weighed Erlenmeyer flask (long narrow form preferable) of $200 \mathrm{cc}$. 
capacity. Saponification was accomplished by adding two cc. of a solution prepared by dissolving fifty grams of potassium hydroxide (free from carbonates) in Ioo cc. of water and five cc. of strong (ninety-five per cent.) alcohol. The flask was connected with a reflux condenser and heated on a water or steambath until saponification was complete. Five minutes was usually amply sufficient for this purpose. The last traces of alcohol were quickly and completely removed with the filter pump by means of a device described by L. F. Nilson. ${ }^{1}$

The soap thus obtained was dissolved in thirty cc. of warm water and decomposed with twenty cc. of a twenty per cent. solution of orthophosphoric acid. The volatile acids were expelled from the flask by means of a current of steam, using the apparatus described below. The operation was usually considered complete when $500 \mathrm{cc}$. had passed over, although, as an extra precaution, fifty cc. more was distilled over and titrated separately. For titration, decinormal soda solution and phenolphthalein were used.

The apparatus used in distilling is shown in the accompanying cut. A is a liter flask two-thirds full of water, which is kept boiling. The steam generated is forced into the decomposed

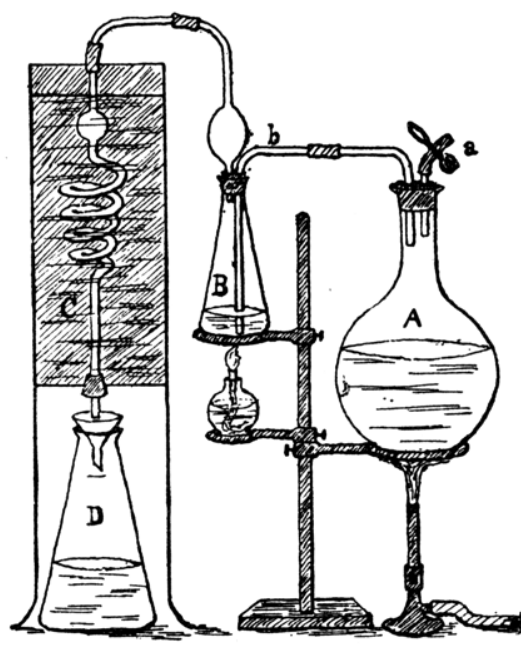
soap solution in B through the tube $b$, which reaches nearly to the bottom of the flask. This second flask is provided with a small lamp, so regulated as to prevent increase, by condensation, of the solution in the flask, and finally to bring its contents to a small bulk (about fifteen cc.). The condenser $\mathrm{C}$ consists of a copper tank, fifteen inches high, twenty inches long, and five inches wide, having four perforations in the bottom, into which glass spirals are fitted by means of rubber stoppers, and provided with proper inlet and 1 Ztschr. anal. Chem., 28, 176. 
overflow pipes for the water supply. Provision was thus made for four distillations at one time. The distillates were collected in wide-mouthed Erlenmeyer flasks, marked on the neck at 500 cc., and carrying funnels with small filters, through which the distillates passed.

Insoluble Acid. - After the distillation was completed, the condenser and connections were rinsed back with boiling water into the flask containing the non-volatile acids. The latter were shaken up with the hot water, and the solution, when cold, was passed through the same filter used for the distillate. Hot water (about $100 \mathrm{cc}$.) was again added, shaken up with the contents of the flask, and when cold filtered off as before. This operation was repeated until the washings contained no trace of phosphoric acid. The filter was then exhausted with hot, strong alcohol, and the solution allowed to run into the flask containing the insoluble acids. The alcohol was driven off on the water-bath and the fatty acids dried at $100^{\circ} \mathrm{C}$. in the air-bath until they began to gain weight.

Very uniform and concordant results were obtained by this method in the examination of a large number of samples of butter fat.

The expulsion of the volatile acids by means of a current of steam, which it was afterwards found ${ }^{1}$ had already been suggested by Goldman, ${ }^{2}$ proved to be a decided improvement over direct boiling of the solution containing the fatty acids. When the operation had been started it required but little further attention and there was no danger of bumping or of overheating the insoluble acids. The volatile acids were very thoroughly removed, the last distillate of fifty cc. never requiring more than two-tenths cc. of soda solution for neutralization.

The first fifty $c c$. of distillate obtained by this method required less soda solution than that obtained by the Reichert or the Moore-Waller methods, and titration of each successive fifty $\mathrm{cc}$. of the total 550 showed that expulsion of the volatile acids was much more gradual and uniform than in the latter methods.

1 Analyst, Sept. 1892,174 .

2 Chem. Ztg., 1888, 12, 183; 14, 216; and 20, 317 . 